\title{
Short Communication: Estimate of Non electrostatic Interaction Free Energy Parameters for Milk Fat Globules
}

\author{
L. J. Kiely* and N. F. Olson ${ }^{\star} \dagger$ \\ *Department of Food Science \\ University of Wisconsin-Madison, Madison 53706 \\ †Wisconsin Center for Dairy Research \\ University of Wisconsin-Madison, Madison 53706
}

\begin{abstract}
The surface free energy components for bovine milk fat globules (MFG) were calculated by solving a modified Young-Good-Fowkes-Girafalco equation with experimentally obtained contact angles for liquids of known surface tension components. The surface free energy of MFG was dominated by the electron donor term $\left(\gamma^{-}\right)$, which formed the basis of a significant repulsive acid-base interaction free energy term at the minimum equilibrium distance. Consideration of the decay of the acid-base and Lifshitz-van der Waals energy term over distance within the context of an "extended DLVO" model shows that physical contact of MFG in the absence of electrostatic repulsion (at their isoelectric point) is prohibited by the acid-base term but that "pseudo-flocculation" might occur at 4- to 5-nm separation in an energy minimum that was $\sim 15 \mathrm{kT}$ for MFG of diameter $=1.0 \mu \mathrm{m}$.
\end{abstract}

(Key words: interaction, milk fat globule, milk)

Abbreviation key: DLVO = Derjaguin Landau Verwey Overbeek, $\mathbf{M F G}=$ milk fat globule.

Native or washed bovine milk fat globules do not aggregate at their isoelectric point of $\mathrm{pH} 3.7$ (Walstra, 1983). Such behavior defies expectations based on Derjaguin Landau Verwey Overbeek (DLVO) theory (Dickinson, 1992), which only allows for a summation of electrostatic repulsive forces and van der Waals attractive forces. Because obviation of the repulsive electrostatic term at the isoelectric point does not cause aggregation of milk fat globules, it may be inferred that some nonelectrostatic force exists that quantitatively exceeds the attractive van der Waals term. Israelachvilli (1991) has described the importance of several non-DLVO forces (solvation, structural, hydration, steric, and fluctua-

Received May 20, 2003.

Accepted July 2, 2003.

Corresponding author: L. J.Kiely; e-mail: kielyj@wyeth.com. tion) in the interaction of surfaces separated by liquid. With respect to milk fat globules (MFG), steric repulsion caused by interpenetration and/or compression of hydrophilic chains that extend from the MFG surface has been implicated but defies easy measurement (Walstra, 1983).

Van Oss (1994) developed an approach to explain particle-particle interaction in aqueous media that may be useful in examining MFG interactions. Fundamental to the approach of van Oss (1994) is the distinction between apolar interactions (Lifshitz-van der Waals interactions consisting of dispersion, orientation, and induction interactions) and polar interactions (electron acceptor (Lewis acid) and electron donor (Lewis base)]. These parameters are experimentally accessible through measurement of the sessile contact angle $(\theta)$ made by polar and nonpolar liquids whose surface tension components are known. It has been shown for a diversity of surfaces [e.g., erythrocytes (van Oss et al., 1972), clay particles (Wu et al., 1994), and bacteria (Kiely and Olson, 2000)] with relatively low zeta $(\zeta)$ potential (say $<-60 \mathrm{mV}$ ) in low ionic strength aqueous environments, that the electron donor parameter $\left(\gamma^{-}\right)$is the principal deterrent to aggregation at the minimum equilibrium distance. Here we report on preliminary observations of the surface free energy parameters of native bovine MFG and explore the implications for MFG interactions as a function of distance.

Milk from three Holstein cows was aspirated from the milk line throughout the entire milking period and immediately washed without cooling by the method of Patton and Huston (1986). This involved the centrifugation of 30 to $40 \mathrm{ml}$ of milk at room temperature for 15 $\min$ at $1700 \times g$. The MFG layer was redispersed in wash solution (PBS: $0.14 M \mathrm{NaCl}$ in $0.01 M$ sodium phosphate buffer, $\mathrm{pH}$ 7.3), and the centrifugation was repeated. This step was repeated two more times. After washing, the globule layer was redispersed in a small volume of wash solution, added drop-wise to cellulose acetate membrane filters $(0.45-\mu \mathrm{m}$ pore size; Millipore Corporation, Burlington, MA), and a gentle vacuum was applied. Globule-coated membranes were placed 
on glass coverslips that were coated with double-sided adhesive tape and dried in air for approximately 30 to $40 \mathrm{~min}$. Contact angles were constant after this duration. The shape of 3 to 5 drops of two polar [water (Milli Q); glycerol; Sigma Chemical Co., St. Louis, MO] liquids and one nonpolar (diiodomethane; J. T. Baker, Inc., Phillipsburg, NJ) liquid on globule-coated membranes was examined using a contact angle meter (Kruss USA, Charlotte, NC). For each wetting liquid, a total of three to four membranes were prepared per milk sample. Contact angles $(\theta)$ were calculated from the height (h) and diameter (d) of drops using $\theta=2 \tan ^{-1} 2 \mathrm{~h} / \mathrm{d}$. The Lifshitz-van der Waals $\left(\gamma^{\mathrm{LW}}\right)$, electron-donor $\left(\gamma^{-}\right)$, and electron-acceptor $\left(\gamma^{+}\right)$components of surface free energy were calculated by solving the following equations of van Oss (1994):

$$
\begin{aligned}
(1+\cos \theta) \gamma_{L}=2\left[\left(\gamma_{s}^{L W} \cdot \gamma_{L}^{L W}\right)^{1 / 2}\right. \\
\quad+\left(\gamma_{s}^{+} \cdot \gamma_{L}\right)^{1 / 2}+\left(\gamma_{s}^{-} \cdot \gamma_{L}^{+}\right)^{1 / 2}
\end{aligned}
$$

where subscripts S and L refer to surface (fat globule) and liquid (wetting), respectively. The total surface free energy $\left(\gamma^{\mathrm{TOT}}\right)$ is given by $\gamma^{\mathrm{TOT}}=\gamma^{\mathrm{LW}}+\gamma^{\mathrm{AB}}$, where $\gamma^{\mathrm{AB}}=$ $2\left(\gamma^{+} \cdot \gamma^{-}\right)^{1 / 2}$.

The value of $\gamma^{\mathrm{LW}}, \gamma^{+}$, and $\gamma^{-}$together with the equivalent values for water, were used to calculate $\Delta \mathrm{G}_{l w w l}^{A B}$, i.e., the acid-base interaction free energy term at the minimum equilibrium distance $\left(\mathrm{d}_{0}=1.57 \times 10^{-10} \mathrm{~m}\right)$ and $\Delta \mathrm{G}_{l w l}^{L W}$, i.e., the Lifshitz-van der Waals interaction free energy term at the minimum equilibrium distance. The distance (d) dependent decay for the acid-base energy term was modeled using the following sphere-sphere function of van Oss (1994):

$$
\Delta \mathrm{G}_{(d)}^{A B}=\pi \mathrm{a}_{\mathrm{p}} \lambda \Delta \mathrm{G}_{l w 1(d 0)}^{A B} \mathrm{e}^{\left[\left(\mathrm{d}_{0}-\mathrm{d}\right) / \lambda\right]}
$$

where $a_{p}$ is the hydrodynamic radius of the fat globule, $\lambda$ is the decay length of water $\left[1.0 \times 10^{-9} \mathrm{~m}\right], \mathrm{d}_{0}$ is the minimum equilibrium distance $\left[1.57 \times 10^{-10} \mathrm{~m}\right]$. The distance (d) dependent decay for the Lifshitz-van der Waals energy term was modeled using the retarded van der Waals function for sphere-sphere interactions described originally by Gregory (1981) and as employed by Grasso and Smets (1998):

$$
\Delta \mathrm{G}_{(d)}^{L W}=-\mathrm{A}_{\mathrm{lwl}} \mathrm{a}_{\mathrm{p}} / 12 \mathrm{~d}\left[1-\left(\mathrm{bd} / \lambda_{0}\right) \ln \left(1+\lambda_{0} / \mathrm{bd}\right)\right],
$$

where $A_{l_{w l}}$ is the Hamaker constant for MFG in aqueous media, $1.3 \times 10^{-20} \mathrm{~J}$ (Walstra and Jenness, 1984), $\lambda_{0}$ is the distance traveled by light during the rotation of a Bohr atom electron $\left(1.7 \times 10^{-7} \mathrm{~m}\right)$, and $\mathrm{b}$ is a retardation correction factor of 5.32 (Grasso and Smets, 1998).

\section{RESULTS AND DISCUSSION}

The surface free energy determinations in these experiments must be regarded as estimates since the globules were air-dried. Such a treatment can be reasonably expected to alter molecular arrangement at the globule membrane surface. The contact angles, surface free energy components and interaction free energy terms (excluding electrostatic) for MFG from fresh milk are in Table 1 and show the MFG surface to be hydrophilic.

Whereas the $\gamma^{-}$term was similar in magnitude to the $\gamma^{\mathrm{LW}}$ term, it far exceeded the $\gamma^{+}$component, i.e., the MFG behaved as electron donors (Lewis base). This results in a significant net positive interaction free energy at the minimum equilibrium distance $\left(\mathrm{d}_{0}\right)$ as $\Delta \mathrm{G}_{\mathrm{lwl}}^{A B}$ is far greater than $\Delta \mathrm{G}_{\mathrm{lwl}}^{L W}$ (where $\Delta \mathrm{G}_{\mathrm{lwl}}^{A B}=$ $-4\left[\left(\gamma_{L}^{+} \cdot \gamma_{L}\right)^{1 / 2}+\left(\gamma_{W}^{+} \cdot \gamma_{W}\right)^{1 / 2}-\left(\gamma_{L}^{+} \cdot \gamma_{W}\right)^{1 / 2}-\left[\left(\gamma_{\bar{L}} \cdot \gamma_{W}^{+}\right)^{1 / 2}\right]\right.$ and $\Delta \mathrm{G}_{\mathrm{lwl}}^{L W}=-2\left[\left(\gamma_{L}^{\mathrm{LW}}\right)^{1 / 2}-\left[\left(\gamma_{W}^{\mathrm{LW}}\right)^{1 / 2}\right]^{2}\right)$. Thus, if electrostatic interaction free energy is ignored, then $\Delta \mathrm{G}_{\mathrm{lwl}(d 0)}^{A B}$ represents a significant energy barrier to physical contact (d $=0$ ) of MFG (Table 1). The $\triangle \mathrm{G}_{\mathrm{lwl}}^{E L E C}$ energy term will of course be additive and provide a further energy barrier to physical contact of MFG.

Interpretation of the relative significance of the different energy terms within the context of any theoretical framework requires reconciliation with certain experimental observations for MFG. Specifically, MFG do not come into physical contact (flocculate) upon removal of the repulsive $\triangle \mathrm{G}_{\mathrm{lwl}}^{E L E C}$, i.e., at their isoelectric point,

Table 1. Sessile contact angles, surface free energy components, and interaction free energy terms for bovine milk fat globules.

\begin{tabular}{lllr}
\hline Contact angle $(\theta)$ & Mean $\pm \mathrm{SD}$ & $\begin{array}{l}\text { Surface free } \\
\text { energy components }\end{array}$ & $\mathrm{mJ} / \mathrm{m}^{2}$ \\
\hline$\theta$ water & $50.1 \pm 3.8$ & $\gamma^{\mathrm{LW}}$ & 36.09 \\
$\theta$ glycerol & $58.8 \pm 15.4$ & $\gamma^{+}$ & 0.04 \\
$\theta$ diiodomethane & $46.7 \pm 2.3$ & $\gamma^{-}$ & 36.94 \\
Interaction free energy components & $\mathrm{mJ} / \mathrm{m}^{2}$ & \\
$\Delta \mathrm{G}_{\mathrm{lll}(d 0)}^{A B}$ & 19.94 & \\
$\Delta \mathrm{G}_{\mathrm{lwl}(d 0)}^{L W}$ & -3.58 & \\
\hline
\end{tabular}




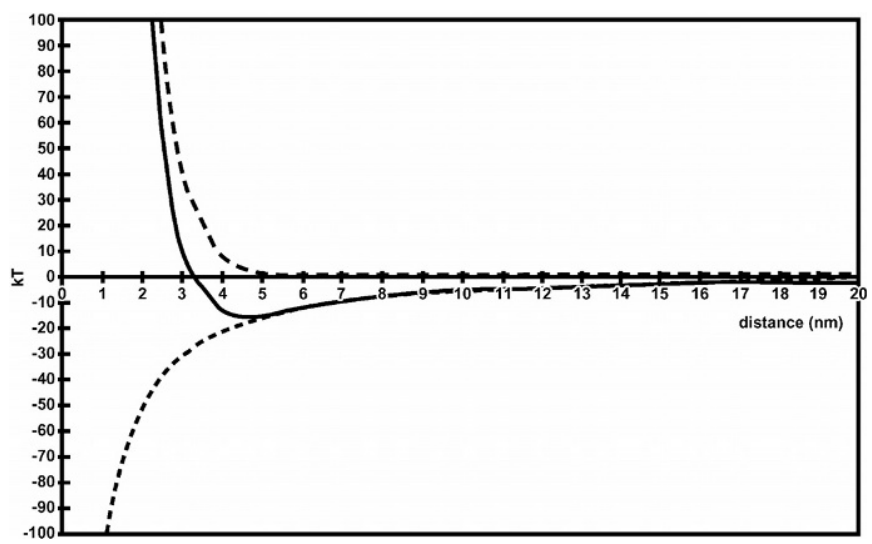

Figure 1. Extended Derjaguin Landau Verwey Overbeek model (minus electrostatic term) for interaction of milk fat globule as a function of distance. Calculations assume Hamaker constant $=1.3 \mathrm{x}$ $10^{-20} \mathrm{~J}$. Acid-base energy (equation 2) is represented by the hatched line (- - -); dispersion energy (equation 3 ) is represented by the dotted line (.....) and the total energy (without electrostatic term) is represented by the solid line.

pH 3.7 (Walstra, 1983). Application of DLVO theory, which sums electrostatic repulsive energy and electrodynamic (van der Waals) attractive energy as a function of distance, to interaction of MFG predicts a net negative interaction energy regardless of MFG diameter or separation distance, which is clearly not the case, i.e., MFG do not spontaneously flocculate (Payens, 1963). The reasons for the failure of the classical DLVO theory to explain MFG interactions are well known and include, among others, an unknown Hamaker constant, lack of a smooth surface, and an unknown surface charge. Meinders et al. (1995) and Grasso and Smets (1998) utilized an extended (to include acid-base interactions) DLVO model (hereafter $\mathrm{DLVO}_{E X}$ ) to describe the distance-dependent interactions of bacteria with other bacteria or with different surfaces. Application of the $\mathrm{DLVO}_{E X}$ model (excluding the electrostatic term) to MFG interaction as a function of distance (by solving equations 2 and 3) results in an interaction energy curve as in Figure 1. A massive energy barrier prevents physical contact of MFG and is attributable to $\Delta \mathrm{G}_{\mathrm{lwl}}^{A B}$. The total energy curve has a primary minimum at 4to 5 -nm separation of close to $15 \mathrm{kT}$, which should be sufficient for "flocculation in the minimum." The depth of the primary minimum deepens for larger globules (data not shown). Critical to the solution of Equation 3 and thereby the dispersion energy curve in Figure 1, is the value of the Hamaker constant $\left(\mathrm{A}_{\mathrm{lwl}}\right)$. Figure 1 reflects the dispersion energy curve for $\mathrm{A}_{\mathrm{lw}}=1.3 \times 10^{-20} \mathrm{~J}$ (Walstra and Jenness, 1984). However, if a lower value of $3.33 \times 10^{-21} \mathrm{~J}$ [employing $\mathrm{A}_{\text {lwl }}=12 \pi \mathrm{d}_{0}^{2} \Delta \mathrm{G}_{(d 0)}^{L W}$ (van Oss, 1994)] is employed for the Hamaker constant, the resultant total interaction energy curve (not shown) is practically positive for all separations and has a minimum at 5- to 7-nm separation of approximately $10 \mathrm{kT}$ for MFG of diameter $=4 \mu \mathrm{m}$.

In conclusion, contact angle measurements on MFG have shown that MFG surfaces behave as electron donors (Lewis base). This results in a significant repulsive energy term $\left(\Delta \mathrm{G}_{\mathrm{lwl}}^{A B}\right)$ that is sufficient to prevent physical contact of MFG even in the absence of electrostatic repulsion. Interpretation of the nonelectrostatic interaction of MFG within the context of an extended DLVO model predicts an energy minimum at 4 - to 5 -nm separation that is deeper than $1.5 \mathrm{kT}$ and therefore would support pseudo-flocculation.

\section{ACKNOWLEDGMENTS}

This work was supported by a gift from Schreiber Foods Inc., Green Bay, WI. Jennifer Butkus, University of Connecticut, provided spreadsheets for calculating the DLVOex functions.

\section{REFERENCES}

Dickinson, E. 1992. Pages 95-97 in An Introduction to Food Colloids. Oxford University Press, New York, NY

Grasso, D., and B. F. Smets. 1998. Equilibrium modeling of pseudomonad aggregation and partitioning to dolomite. J. Disper. Sci. Technol. 19:1081-1106.

Gregory, J. 1981. Approximate expressions for retarded van der Waals interaction. J. Coll. Inter. Sci. 83:138-145.

Israelachvilli, J. N. 1991. Pages 260-311 in Intermolecular and Surface Forces. Academic Press Inc., San Diego, CA.

Kiely, L.J., and N. F. Olson. 2000. The physicochemical surface characteristics of Lactobacillus casei. Food Microbiol. 17:277-291.

Meinders, J. M., H. C. van der Mei, and H. J. Busscher. 1995. Deposition efficiency and reversibility of bacterial adhesion under flow. J. Colloid Interface Sci. 176:329-341.

Patton, S., and G. E. Huston. 1986. A method for isolation of milk fat globules. Lipids 21:170-174.

Payens, T. A. J. 1963. Influence of particle size on the agglutination of milk fat. Neth. Milk Dairy J. 17:150-153.

Van Oss, C. J., R. M. Fike, R. J. Good, and J. M. Reining. 1972. Cell microelectrophoresis simplified by the reduction and uniformization of the electroosmotic backflow. Analyt. Biochem. 60:242-251.

Van Oss, C. J. 1994. Pages 7-45, 75, 80 in Interfacial Forces in Aqueous Media. Marcel Dekker, Inc., New York, NY.

Walstra, P. 1983. Physical chemistry of milk fat globules. Page 128 in Developments in Dairy Chemistry. 2. P. F. Fox, ed. Applied Science Publishers, New York, NY.

Walstra, P., and R. Jenness. 1984. Page 222 in Dairy Chemistry and Physics, John Wiley and Sons, New York, NY.

Wu, W., R. F. Giese, and C. J. van Oss. 1994. Linkage between $\zeta$-potential and electron donicity of charged polar surfaces. 1. Implications for the mechanism of flocculation of particle suspensions with plurivalent counterions. Coll. Surf. A: Physico. Eng. Asp. 89:241-252. 